\title{
Modified rives-stoppa repair for abdominal incisional hernias
}

Peter Nau, Clancy J. Clark, Mason Fisher, Gregory Walker, Bradley J. Needleman, E. Christopher Ellison, Peter Muscarella

Department of Surgery, The Ohio State University, Columbus, America; Pete.Muscarella@osumc.edu

Received 26 October 2009, revised 23 December 2009; accepted 24 December 2009.

\section{ABSTRACT}

Incisional hernias are a prevalent problem in abdominal surgery and occur in $11 \%$ of patients who undergo laparotomy. Primary suture closure of incisional hernias results in a $31 \%-58 \%$ chance of recurrence. The addition of a prosthetic mesh implant decreases recurrence rates to $8 \%-10 \%$. Popularized in Europe by Rives and Stoppa, the sublay technique has proven to be very effective, with low recurrence rates (0\%-23\%) and minimal complications. The purpose of the study was to evaluate the experience of a single surgeon at a large tertiary care center performing a modified Rives-Stoppa repair for abdominal incisional hernias. To do this, the records of all patients undergoing a modified Rives-Stoppa incisional hernia repair between January 2000 and August 2003 were retrospectively reviewed. Outpatient clinic notes, discharge summaries, operative reports, and laboratory data were reviewed for patient demographics, surgical data and postoperative complications. Univariate analysis was performed in order to identify predictors for recurrence. During the study period, 83 patients underwent a modified Rives-Stoppa incisional hernia repair. Nineteen patients were excluded due to incomplete medical records. No patients required postoperative exploration for an intra-abdominal catastrophe. Twenty-five percent $(n=16)$ of patients had a complication as a result of the hernia repair. Only two patients (3.1\%) developed recurrent incisional hernias. History of diabetes $(p=0.007)$ and benign prostatic hyperplasia $(p=0.000)$ were the only significant predictors for recurrence. The results presented here confirm that the modified Rives-Stoppa retromuscular repair is an effective method for the repair of incisional hernias. The complica- tion and recurrence rates compare favorably to results for currently popular alternative techniques.

Keywords: Incisional Hernia Repair; Mesh; Rives-Stoppa Repair; Abdominal Wall Defects

\section{INTRODUCTION}

Incisional hernias are a common problem in abdominal surgery and occur in up to $11 \%$ of patients who undergo laparotomy [1]. Complications of incisional hernias include infection, ulceration, incarceration of viscera, and small bowel obstruction [2-4]. Patients also experience discomfort and a cosmetically unpleasing bulge at the incision site. Successful repair of incisional hernias continues to be challenging. Primary suture closure of incisional hernias results in recurrence rates of 31\%-58\% [5-10]. The addition of prosthetic mesh implants has been shown to decrease the incidence of recurrence to 8\%-10\% [11-14].

A tension-free prosthetic mesh repair of incisional hernias dates back the to 1940's and 1950's with the introduction of metal wire mesh and polypropylene mesh respectively [15-17]. With the development of new prosthetic materials, advances in minimally invasive techniques, and improvements in open surgical procedures, surgeons continue to debate the appropriate operative technique for the repair of incisional hernias, particularly regarding the anatomic placement and type of prosthetic mesh. Various operative techniques for incisional hernia repair use onlay, sublay (retromuscular or extrafascial), or underlay (intraperitoneal or subfascial) placement of mesh. Popularized in Europe by Rives and Stoppa, the sublay technique has proven to be very effective, with low recurrence rates (0\%-23\%) and minimal complications [18-25]. Disadvantages include complexity, long operative times, and the possibility of chronic abdominal pain [26].

The experience of one surgeon at The Ohio State University Medical Center suggests that a modified 
Rives-Stoppa incisional hernia repair compares favorably to the standard Rives-Stoppa repair as well as to other techniques for addressing incisional hernias. The purpose of this study was to fully characterize the complications and recurrence rates of this surgical technique by conducting a retrospective review of patients who had undergone a modified Rives-Stoppa incisional hernia repair.

\section{MATERIALS AND METHODS}

Between January 2000 and August 2003, 83 patients in the practice of one surgeon at a large urban academic hospital (The Ohio State University Medical Center) underwent a modified Rives-Stoppa incisional hernia repair. Of the 83 patients initially identified, 19 patients were excluded due to incomplete medical records. Outpatient clinic notes, discharge summaries, operative reports, and laboratory data of 64 patients were reviewed. There were 20 males and 44 females (mean age 50 years, range 27-85). The majority of incisional hernias were midline and supraumbilical, with several $(n=3)$ flank hernias. Forty-five percent $(n=29)$ of the incisional hernias were recurrent (mean 2, range 1-7, S.D. \pm 1.6). At the time of repair, most incisional hernias were symptomatic and evident on physical exam. Six percent $(n=4)$ were incarcerated at the time of presentation and one patient had an infected abdominal wound (Table 1).

The operative technique is a variation of the previously described Rives-Stoppa technique [18,22,27]. All patients received intravenous prophylactic antibiotics (Cefazolin, $1 \mathrm{~g}$ ) prior to incision. Patients were placed supine for midline hernias, or in lateral decubitus for flank hernias. The old incision scar and hernia sac were removed en bloc using an elliptical incision. Dissection of the hernia sac in all patients required entry into the peritoneum and division of adhesions to the sac, if identified. The fascial plane between the rectus muscle and posterior rectus sheath was dissected as far lateral as possible, typically 5-10 cm, between eight and ten polybutester (Novofil, United States Surgical/Syneture, Norwalk, CT) "U-stitch” anchoring sutures were placed only in the posterior rectus sheath, not penetrating the rectus muscle, anterior rectus sheath or skin. The fascial defect was closed by reapproximating the posterior rectus sheath with running polybutester suture reinforced with simple interrupted polybutester suture (Figure 1). Tension was minimal and the posterior rectus fascia was closed in all 64 patients. Below the arcuate line, the peritoneum was carefully reapproximated. Monofilament polypropylene mesh (Bard Mesh Flat Sheets, Davol Inc., Cranston, RI) was cut to size slightly larger (5 cm overlap) than the fascial defect and anchored using the previously placed sutures (Figure 2). Cefazolin (1 g) powder was placed on the mesh prior to closure of
Table 1. Hernia characteristics.

\begin{tabular}{cc}
\hline Recurrent & $\mathrm{N}(\%)$ \\
Location & $29(45.3)$ \\
Supraumbilical & $42(65.6)$ \\
Umbilical & $9(14.1)$ \\
Infraumbilical & $8(12.5)$ \\
Paramedian & $1(1.6)$ \\
Subcostal & $1(1.6)$ \\
Flank & $3(4.7)$ \\
Symptomatic & $59(92.2)$ \\
Evident on Physical Exam & $61(95.3)$ \\
Incarcerated & $4(6.4)$ \\
Infected & $1(1.6)$ \\
Ulcerated & $0(0)$ \\
\hline
\end{tabular}

the anterior rectus sheath (Figure 3). In the majority of the patients $(n=46)$, one to two bulb suction drains were placed between the skin and the anterior rectus sheath. The skin was approximated with interrupted, dermal 2-0 polyglycolic acid sutures (Dexon II, Syneture, Norwalk, CT) and staples. Oral prohylactic antibiotics (Cefalexin, 500 mg PO TID) were started postoperatively and continued until drain removal. Hospital discharge typically occurred on post-operative day 3 (mean 5, range 2-29, S.D. \pm 3.9).

Follow-up information was available for 61 patients, with mean follow-up of 20 weeks (range 1-144 weeks, S.D. \pm 30.1) and mean number of follow-up visits of three (range 1-13, S.D. \pm 2.9). All patients were examined during follow-up by the surgeon who completed the operation. Three patients had no follow-up information available and their records indicated that another physician is currently following them. Physician correspondence information was reviewed to determine any complications or recurrences in these three patients. One patient died of causes unrelated to the repair of the incisional hernia, and there is no evidence that any of these patients developed a recurrent incisional hernia. This study was approved by the medical center institutional review board. Statistical analysis was conducted using SPSS for Windows (Version 11.5.0, SPSS Inc., Chicago, IL).

\section{RESULTS}

All 64 patients tolerated the procedure well with no intraoperative complications. There were two (3.1\%) perioperative complications. No myocardial infarctions or cases of pneumonia were recorded. One patient suffered a pulmonary embolism and was treated with anticoagulant therapy. The second patient was readmitted for drainage of a rectus sheath hematoma found anterior to the prosthetic mesh. Three $(4.7 \%)$ patients required perioperative packed red blood cell transfusions. Blood loss was negligible, with a mean post-operative decrease in hemoglobin of $0.8 \mathrm{~g} / \mathrm{dl}$ (S.D. \pm 1.3 ). 


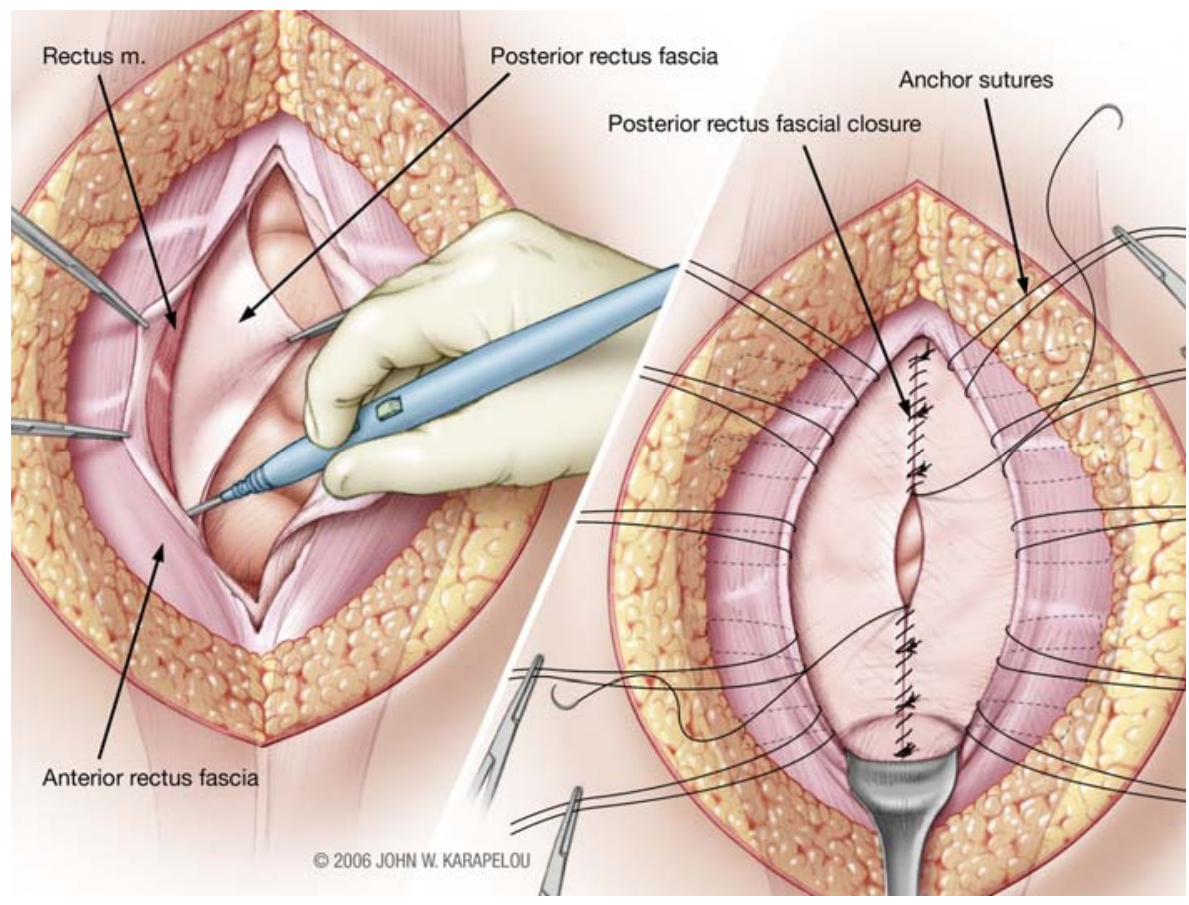

Figure 1. The posterior rectus sheath is dissected away from the overlying rectus abdominus muscle on both sides of the defect using electrocautery. The peritoneum is mobilized below the level of the arcuate line. Care must be taken to ligate and divide perforating blood vessels, as unidentified injury to these structures could result in the formation of rectus hematomas postoperatively. The posterior layer is approximated with running suture and reinforced intermittently with additional suture in order to avoid excess tension and tearing during manipulation.

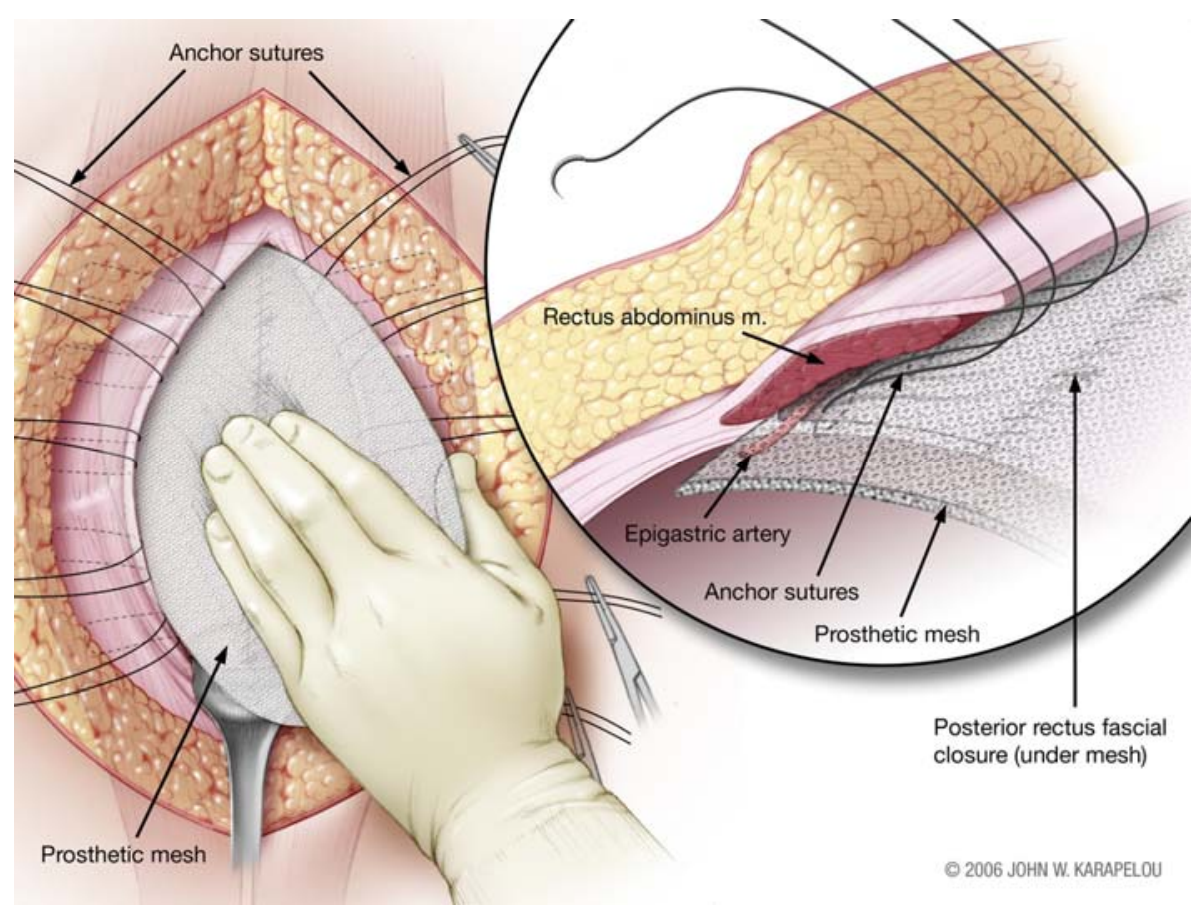

Figure 2. Polypropylene mesh is trimmed and placed into the space behind the rectus muscle. Previously placed anchoring sutures are passed through the mesh and used for fixation. 


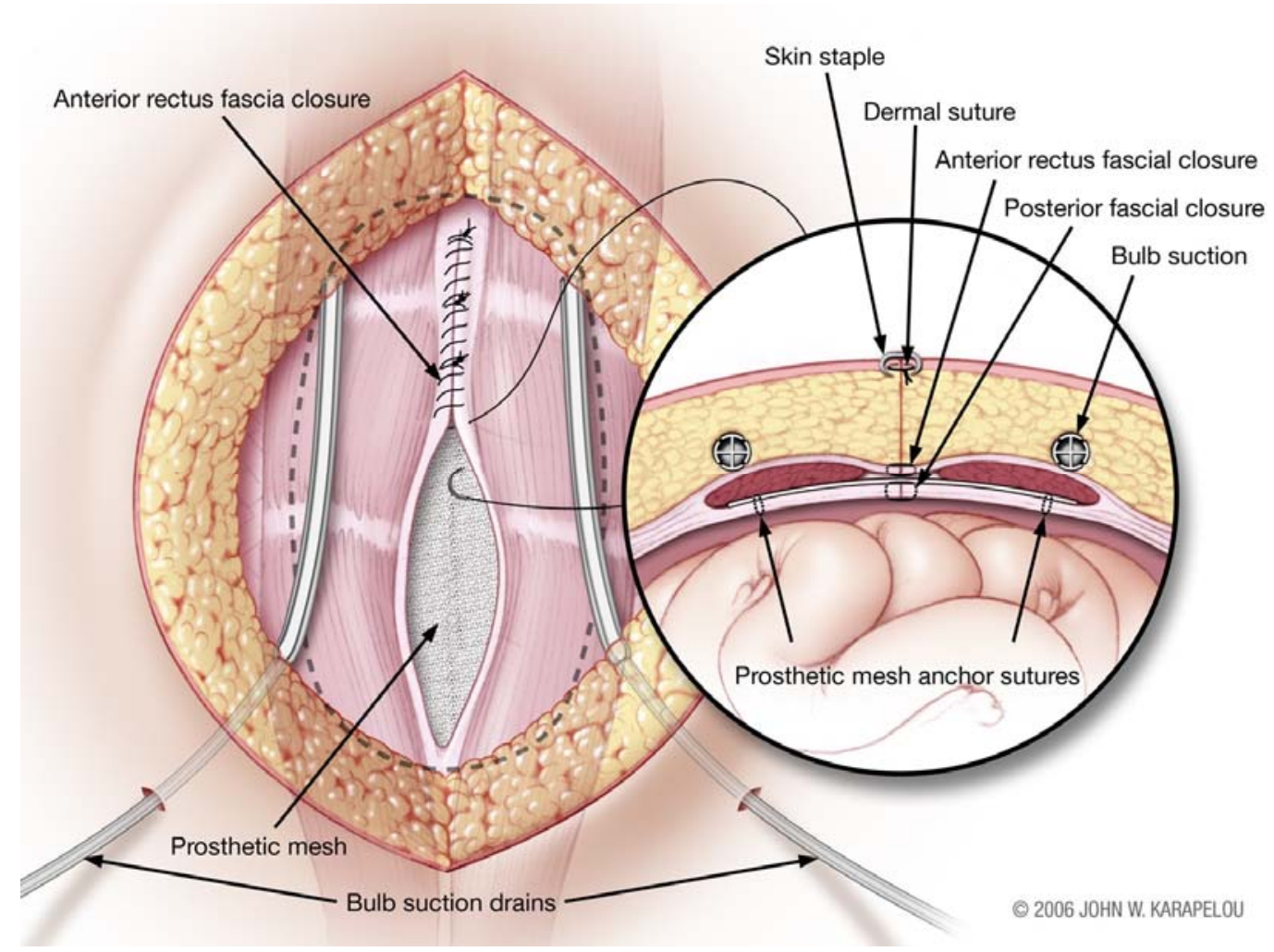

Figure 3. Bulb suction drains are placed into the subcutaneous space and brought out of the skin through separate stab incisions. The skin is approximated with dermal sutures and staples in order to maintain an adequate seal for drain.

There were complications in twenty-five percent $(n=16)$ of patients as a result of the hernia repair. The majority of the complications were minor with only seven patients requiring admission to the hospital for management (15.6\%). Six patients $(9.4 \%)$ had superficial wound or deep mesh infections, defined as purulent drainage or positive wound cultures. None required removal of the prosthetic mesh and all were successfully managed with antibiotics and wound management. One patient developed erythema adjacent to the skin staples, four $(6.3 \%)$ developed seromas, and three $(4.7 \%)$ developed wound hematomas. In no case was evidence of infection, including erythema or purulent drainage noted in those patients with seromas. Wound cultures were not performed routinely on suspected seromas in the absence of clinical signs of infection. A hematoma was defined as a fluid collection with bloody drainage. Although no patients developed fistulas, four patients (6.3\%) presented post-operatively with partial small bowel obstructions, all of who were successfully treated with nasogastric decompression. Fifteen patients (23.4\%) are being managed for chronic abdominal pain (Table 2). In the subgroup of morbidly obese patients $(n=27)$, there were two partial small bowel obstructions (7.4\%), one seroma (3.7\%), one hematoma (3.7\%), and two surgical site in-
Table 2. Complications.

\begin{tabular}{cc}
\hline & $\mathrm{N}(\%)$ \\
Infection & $6(9.4)$ \\
Seroma & $4(6.3)$ \\
Hematoma $^{*}$ pSBO $^{*}$ & $3(4.7)$ \\
Staple Reaction & $4(6.3)$ \\
Fistula & $1(1.6)$ \\
Chronic Pain & $0(0)$ \\
Recurrence & $15(23.4)$ \\
\hline
\end{tabular}

*partial small bowel obstruction.

fections (7.4\%).Two patients (3.1\%) developed recurrent incisional hernias. The first patient developed a small $(1-2 \mathrm{~cm})$ supraumbilical recurrent hernia 10 months (post-operative day 285) following the hernia repair. The patient subsequently developed a second small periumbilical hernia. The second patient was originally treated for a recurrent midline incisional hernia and a primary flank hernia. Recurrence of the flank hernia developed six weeks post-operatively (post-operative day 42). The flank hernia has recurred twice and it is believed that inadequate repair can be attributed to insufficient overlap of mesh and fascia or incomplete coverage of the fascial defect. Both patients had medical histories significant for diabetes and a respiratory disorder. The study population was a typical patient population for recurrent complex 
Table 3. Potential prognostic factors for hernia recurrence.

\begin{tabular}{lll}
\hline & $\mathrm{N}(\%)$ & $\mathrm{p}$ \\
Smoking & $18(28.1)$ & \\
COPD $^{*}$ & $8(12.5)$. & 0.103 \\
Asthma & $7(10.9)$ & 0.072 \\
Diabetes (Type I or II) & $14(21.9)$ & 0.007 \\
Sleep Apnea & $12(18.8)$ & 0.490 \\
Morbid Obesity & $27(42.2)$ & 0.220 \\
Chronic Steroid Use & $3(4.7)$ & 0.750 \\
Benign Prostatic Hyperplasia & $2(3.1)$ & 0.000 \\
Cirrhosis & $3(4.7)$ & 0.750 \\
Malabsorptive Surgery $^{\dagger}$ & $30(46.9)$ & 0.177 \\
\hline
\end{tabular}

*COPD, chronic obstructive pulmonary disease. †Malabsorptive surgery defined as roux-en-Y gastric bypass (25), cystojejunostomy (1), choledochojejunostomy (3), or vertical banded gastroplasty (1).

incisional hernias, with numerous potential risk factors for recurrence (Table 3). Forty-two percent of patients $(n=27)$ were considered to be morbidly obese (BMI $>40$ $\mathrm{kg} / \mathrm{m} 2)$ and $46.9 \%$ of patients $(\mathrm{n}=30)$ had previously undergone a surgical procedure that could potentially result in malabsorption (Rouxen-Y gastric bypass, choledocho-jejunostomy, etc.). In the subgroup of morbidly obese patients, no recurrences were observed. With univariate analysis, a history of diabetes $(\mathrm{p}=0.007)$ or benign prostatic hyperplasia $(\mathrm{p}=0.000)$ were the only significant prognostic factors for recurrence.

\section{DISCUSSION}

Surgical techniques for the repair of incisional hernias continue to evolve with advances in prosthetic materials and minimally invasive technology. Luijendijk et al. conducted a randomized, multicenter study suggesting that mesh repair is superior to suture repair [28]. The optimal technique for mesh placement has not been established and remains controversial. Laparoscopic repair appears to have numerous benefits, including low recurrence rates, decreased hospital length of stay, decreased postoperative pain, and earlier return to work and normal activities. Although a single randomized trial has been designed [29], the results are still pending and no randomized controlled trials are available to prove any benefit of laparoscopic techniques over open repair. A meta-analysis by Goodney et al. indicated that laparoscopic ventral hernia repair may have lower complication rates [30]. Initial concern for increased fistula formation and small bowel obstruction with the laparoscopic underlay techniques have also been raised, but appear to be less of concern with recent studies and changes in mesh technology [11]. It has been our experience that catastrophic abdominal complications, such as small bowel perforation, can occur with the laparoscopic repair. Although this does not appear to occur frequently, these types of complications can be disastrous in that they may require multiple surgeries, mesh removal, long-term wound care, and skin grafting procedures. The end result is usually an incisional hernia that is larger than the initial hernia. There are currently no reliable methods for selecting appropriate patients for laparoscopic incisional hernia repair. The data from our study suggests that the technique described here is rarely, if ever, associated with these types of complications.

The separation of components technique, originally described by Ramirez et al. in 1990 has recently drawn interest for complex incisional hernia repair. After mobilization of skin flaps, the external oblique muscle is released just lateral to its rectus sheath insertion from the underlying internal oblique muscle. This enables the well vascularized rectus abdominis muscle complex to be advanced medially, allowing for coverage of the hernia defect [31]. Up to a $20 \mathrm{~cm}$ defect at the waist, $12 \mathrm{~cm}$ in the upper abdomen, and $10 \mathrm{~cm}$ in the lower abdomen can be closed using additional relaxing incisions. Advantages include utilization of a dynamic muscle group that remains innervated for closure of the defect, and potentially improved cosmetic results as excess skin is commonly excised prior to closure of the wound. Shestak et al. reported only one recurrence, two superficial wound infections, and one seroma in 22 patients undergoing this procedure [32]. In contrast, another study demonstrated recurrent hernias in $32.0 \%$ of patients and complications, including fascial dehiscence, hematoma, seroma, wound infection, skin necrosis, and respiratory insufficiency, in $39.5 \%$ of patients [33]. The modified Rives-Stoppa technique allows for cosmetically pleasing results, in that redundant skin is removed en bloc with the underlying hernia sac. Skin necrosis is rare because large skins flaps are not created and careful attention is paid to preserving the perforating blood vessels that supply the remaining skin and subcutaneous tissues. The Rives-Stoppa technique preserves the functionality and integrity of the abdominal wall, factors considered to be crucial for effective repair of abdominal wall defects by proponents of the separation of components technique. Furthermore, the Rives-Stoppa technique employs the use of mesh as reinforcement, and this is generally believed to decrease recurrence rates as described previously.

The data reported here indicate that a modified Rives-Stoppa retromuscular repair results in favorable recurrence and complication rates when compared with the standard Rives-Stoppa repair as well as other techniques. The experience of a single surgeon's practice at The Ohio State University is comparable to previously reported results (Table 4). Benefits of this technique include the ability to explore the entire fascial defect and to identify any potentially weak points in the fascia. Fenestrations in the peritoneum can be recognized, and if necessary, selection of a larger mesh implant to cover 
Table 4. Studies of the rives-stoppa open mesh repair

\begin{tabular}{cccc}
\hline & $\begin{array}{c}\text { Seroma, } \\
\mathrm{N}(\%)\end{array}$ & $\begin{array}{c}\text { Infection, } \\
\mathrm{N}(\%)\end{array}$ & $\begin{array}{c}\text { Recur- } \\
\text { rence, N(\%) }\end{array}$ \\
\hline Knight et al. $2002[18]$ & $1(1.5)$ & $2(3.1)$ & $0(0)$ \\
Bauer et al. $2002[19]$ & $7(12.3)$ & $2(3.5)$ & $0(0)$ \\
Toniato et al. $2002[22]$ & -- & $6(7.8)$ & $2(2.6)$ \\
Luijendijk et al. $2000[21]$ & $4(4.8)$ & $3(4)$ & $17(23)$ \\
Balen et al. 1998 [26] & $2(4.4)$ & $1(2)$ & $1(2)$ \\
McLanahan et al. $1997[24]$ & $1(1)$ & $13(13)$ & $3(3.5)$ \\
Sugerman et al. $1996[23]$ & $5(5)$ & $17(17)$ & $4(4)$ \\
Temudom et al. $1996[28]$ & $3(6)$ & $6(12)$ & $2(4)$ \\
Adloff et al. 1987 [30] & -- & $3(2.3)$ & $6(4)$ \\
\hline
\end{tabular}

these areas of potential weakness can be made. Placement of the mesh between the posterior rectus sheath and the rectus muscle takes advantage of the intra-abdominal pressure to secure the mesh, while minimizing the risk of adhesion and fistula formation. Unique to this series of patients was the surgeon's ability to close the posterior rectus sheath with suture. Extensive dissection of the plane between the posterior rectus sheath and the rectus muscle likely decreases tension when closing this layer. Inability to close the posterior rectus sheath requires bridging the fascial defect and would likely increase the risk of recurrence.

It should be noted that the majority of the complications in this series were minor and easily managed on an outpatient basis. Despite pre- and post-operative prophylactic antibiotics, bulb suction drainage, and direct placement of cefazolin powder on the prosthetic mesh, the infection rate still approached $10 \%$. This may be attributed to patient factors, natural reaction to a foreign material, and/or long-term placement of bulb suction drains. Colonization of drain sites increases with time and they are a potential portal of entry for bacterial infection [34]. Criteria for drain removal generally required that the drain output be less than $30 \mathrm{ml}$ per day in an attempt to minimize the incidence of seroma formation. As a result, drains remained in place for a mean duration of three weeks (range 7-85 days, S.D. \pm 15.7 ). Removing drains earlier might help to decrease infection rates. It is also unclear whether continuing oral antibiotics while the drains are in imparts any benefit for the patient, but was performed in order to decrease the chance of infection. The direct application of antibiotics on the mesh at the time of surgery is clearly controversial, but has been described previously $[35,36]$. The rationale is to improve the local concentration of antibiotics and this may have potential benefits, particularly for obese patients in whom systemic antibiotic therapy may not be as effective. Although post-operative complications were not significant risk factors for hernia recurrence (data not shown), other studies have recognized an increased risk of recurrence following infection [28,37]. The concern is generally that infected mesh must be re- moved to successfully treat the infection and removal of the mesh results in hernia recurrence. In a study of patients undergoing concurrent incisional hernia repair and elective colon resections, the authors concluded that prosthetic mesh may be employed for incisional hernia repairs in contaminated fields without increasing the risk of complications [38]. In a study comparing different mesh materials, Leber et al. concluded that multifilament polyester mesh (Mersilene) has a significantly increased risk of infection as compared to double-filament polypropylene mesh (Prolene) [39]. Infection rates vary among studies, but this is understandable given variations in antibiotic usage, drain management, and types of mesh employed. Considering the current body of evidence, the appropriate prevention and management of wound infection in patients undergoing mesh incisional hernia repair remains to be determined.

Chronic pain was a concern of almost a quarter of study patients, but comparison of pre and post-operative pain was not available in this study. The concern of chronic pain has also been raised in previous studies [12, 20]. McLanahan et al. reported that $11 \%$ of patients had moderate to severe pain at 12 months after incisional hernia repair [20]. In a 1997 symposium on incisional hernia repair, Schumpelick argued that mesh can limit range of motion and result in a stiff abdomen [21]. Decreased abdominal wall compliance has been confirmed with three dimensional stereography [40]. Given the potentially negative long-term effects of prosthetic mesh repair, data characterizing the quality-of-life, chronic pain, and physical limitations of mesh implants should prove to be helpful.

Although diabetes and benign prostatic hyperplasia were the only identified risk factors for recurrence in this study, previously identified risk factors for recurrence were common in the study population. Considering the size and non-randomized nature of this study, it is possible that we did not have sufficient statistical power to display the significance of these known risk factors. Sugerman et al. have shown that severe obesity is a greater risk factor for hernia recurrence than chronic steroid use [41]. In the subgroup of morbidly obese patients we expected to see increased rates of recurrence and infection, however, this was not the case. The majority of the obese patients who underwent hernia repair were actively losing weight from recent gastric bypass surgery. It is possible that weight loss, the presence of redundant skin, and general changes in body habitus facilitated the repair and decreased the risk of recurrence.

The modified Rives-Stoppa retromuscular repair, as described here, appears to be an effective treatment for incisional hernias. These results compare favorably with other published reports for the Rives-Stoppa repair and other techniques. The recurrence rate of $3.2 \%$ is clearly 
acceptable in this series that includes numerous patients with multiple risk factors, and a considerable number of patients who have failed previous attempts at repair. We also believe that the absence of catastrophic abdominal events following repair is important to note. Wound infection, chronic pain, and persistent abdominal stiffness continue to be problematic, but manageable. Randomized prospective trials are required to determine the optimum technique for incisional hernia repair.

\section{REFERENCES}

[1] Mudge, M. and Hughes, L.E. (1985) Incisional hernia: a 10 year prospective study of incidence and attitudes. $\mathrm{Br} \mathrm{J}$ Surg, 72, 70-71.

[2] Petersen, S., Henke, G.., Freitag, M., Faulhaber, A. and Ludwig, K. (2001) Deep prosthesis infection in incisional hernia repair: predictive factors and clinical outcome. Eur J Surg, 167, 453-457.

[3] Read, R.C., and Yoder, G. (1989) Recent trends in the management of incisional herniation. Arch Surg, 124, 485-488.

[4] Rives, J., Lardennois, B., Pire, J.C. and Hibon, J. (1973) [Large incisional hernias. The importance of flail abdomen and of subsequent respiratory disorders]. Chirurgie, 99, 547-563.

[5] George, C.D. and Ellis, H. (1986) The results of incisional hernia repair: a twelve year review. Ann $R$ Coll Surg Engl, 68, 185-187.

[6] Langer, S. and Christiansen, J. (1985) Long-term results after incisional hernia repair. Acta Chir Scand, 151, 217-219.

[7] Luijendijk, R.W., Lemmen, M.H., Hop, W.C. and Wereldsma, J.C. (1997) Incisional hernia recurrence following "vest- over-pants" or vertical Mayo repair of primary hernias of the midline. World J Surg, discussion 66, 21, 62-65.

[8] Manninen, M.J., Lavonius, M. and Perhoniemi, V.J. (1991) Results of incisional hernia repair. A retrospective study of 172 unselected hernioplasties. Eur J Surg, 157, 29-31.

[9] Stevick, C.A., Long, J.B., Jamasbi, B. and Nash, M. (1988) Ventral hernia following abdominal aortic reconstruction, Am Surg, 54,287-289.

[10] van der Linden, F.T. and van Vroonhoven, T.J. (1988) Long-term results after surgical correction of incisional hernia, Neth J Surg, 40, 127-129.

[11] Cassar, K. and Munro, A. (2002) Surgical treatment of incisional hernia, Br J Surg, 89, 534-545.

[12] Korenkov, M., Sauerland, S., Arndt, M., Bograd, L., Neugebauer, E.A. and Troidl, H. (2002) Randomized clinical trial of suture repair, polypropylene mesh or autodermal hernioplasty for incisional hernia, $\mathrm{Br} J$ Surg, 89, 50-56.

[13] Liakakos, T., Karanikas, I., Panagiotidis, H. and Dendrinos, S. (1994) Use of Marlex mesh in the repair of recurrent incisional hernia. Br J Surg, 81, 248-249.

[14] Molloy, R.G., Moran, K.T., Waldron, R.P., Brady, M.P. and Kirwan, W.O. (1991) Massive incisional hernia: ab- dominal wall replacement with Marlex mesh. Br J Surg, 78, 242-244.

[15] Crosthwait, R.W. (1960) Ventral hernia: a rapid method of repair. Am J Surg. 99, 330-331.

[16] Usher, F.C., Ochsner, J. and Tuttle Jr., L.L. (1958) Use of marlex mesh in the repair of incisional hernias. Am Surg, 24, 969-974.

[17] Validire, J., Imbaud, P., Dutet, D. and Duron, J.J. (1986) Large abdominal incisional hernias: repair by fascial approximation reinforced with a stainless steel mesh. $\mathrm{Br} J$ Surg, 73, 8-10.

[18] Bauer, J.J., Harris, M.T., Gorfine, S.R. and Kreel, I. (2002) Rives-Stoppa procedure for repair of large incisional hernias: experience with 57 patients. Hernia, 6, 120-123.

[19] Knight, R. and Fenoglio, M.E. (2002) The use of the Kugel mesh in ventral hernia repairs. Am J Surg, 183, 642-645.

[20] McLanahan, D., King, L.T., Weems, C., Novotney, M. and Gibson, K. (1997) Retrorectus prosthetic mesh repair of midline abdominal hernia. Am J Surg, 173, 445-449.

[21] Schumpelick, V., Junge, K., Rosch, R., Klinge, U. and Stumpf, M. (2002) [Retromuscular mesh repair for ventral incision hernia in Germany]. Chirurg, 73, 888-894.

[22] Stoppa, R.E. (1989) The treatment of complicated groin and incisional hernias. World J Surg, 13, 545-554.

[23] Temudom, T., Siadati, M. and Sarr, M.G. (1996) Repair of complex giant or recurrent ventral hernias by using tension-free intraparietal prosthetic mesh (Stoppa technique): lessons learned from our initial experience (fifty patients). Surgery, discussion 743-734,120, 738-743;

[24] Toniato, A., Pagetta, C., Bernante, P., Piotto, A. and Pelizzo, M.R. (2002) Incisional hernia treatment with progressive pneumoperitoneum and retromuscular prosthetic hernioplasty. Langenbecks Arch Surg, 387, 246-248.

[25] Yaghoobi Notash, A., Yaghoobi Notash, A., Jr., Seied Farshi, J., Ahmadi Amoli, H., Salimi, J. and Mamarabadi, M. (2006) Outcomes of the Rives-Stoppa technique in incisional hernia repair: ten years of experience. Hernia.

[26] LeBlanc, K.A. and Whitaker, J.M. (2002) Management of chronic postoperative pain following incisional hernia repair with Composix mesh: a report of two cases. Hernia, 6, 194-197.

[27] Rives, J. (1987) Major incisional hernia. In J. P. Chevrel (Ed.), Surgery of the abdominal wall, New York: Springer-Verlag, 116-144.

[28] Luijendijk, R.W., Hop, W.C., van den Tol, M.P., de Lange, D.C., Braaksma, M.M., JN, I.J., Boelhouwer, R. U., de Vries, B.C., Salu, M.K., Wereldsma, J.C., Bruijninckx, C.M. and Jeekel, J.A. (2000) Comparison of suture repair with mesh repair for incisional hernia. $N$ Engl J Med, 343, 392-398.

[29] Itani, K.M., Neumayer, L., Reda, D., Kim, L. and Anthony, T. (2004) Repair of ventral incisional hernia: the design of a randomized trial to compare open and laparoscopic surgical techniques. Am J Surg, 188, 22S-29S

[30] Goodney, P.P., Birkmeyer, C.M. and Birkmeyer, J.D. (2002) Short-term outcomes of laparoscopic and open ventral hernia repair: a meta-analysis. Arch Surg, 137, 1161-165. 
[31] Ramirez, O.M., Ruas, E. and Dellon, A.L. (1990) "Components separation" method for closure of abdominal-wall defects: an anatomic and clinical study. Plast Reconstr urg, 86, 519-526.

[32] Shestak, K.C., Edington, H.J. and Johnson, R.R. (2000) The separation of anatomic components technique for the reconstruction of massive midline abdominal wall defects: anatomy, surgical technique, applications, and limitations revisited. Plast Reconstr Surg, quiz 739, 105, 731-738.

[33] de Vries Reilingh, T.S., van Goor, H., Rosman, C., Bemelmans, M.H., de Jong, D., van Nieuwenhoven, E.J., van Engeland, M.I. and Bleichrodt, R.P. (2003) "Components separation technique" for the repair of large abdominal wall hernias. J Am Coll Surg, 196, 32-37.

[34] Drinkwater, C.J. and Neil, M.J. (1995) Optimal timing of wound drain removal following total joint arthroplasty. $J$ Arthroplasty, 10, 185-189.

[35] Dirschl, D.R., and Wilson, F.C. (1991) Topical antibiotic irrigation in the prophylaxis of operative wound infections in orthopedic surgery. Orthop Clin North Am, 22, 419-426.

[36] O'Connor Jr, L.T. and Goldstein, M. (2002) Topical perioperative antibiotic prophylaxis for minor clean inguinal surgery. J Am Coll Surg, 194, 407-410.

[37] Bauer, J.J., Harris, M.T., Kreel, I. and Gelernt, I.M. (1999) Twelve-year experience with expanded polytetrafluoroethylene in the repair of abdominal wall defects.
Mt Sinai J Med, 66, 20-25.

[38] Birolini, C., Utiyama, E.M., Rodrigues Jr., A.J. and Birolini, D. (2000) Elective colonic operation and prosthetic repair of incisional hernia: does contamination contraindicate abdominal wall prosthesis use? J Am Coll Surg, 191, 366-372.

[39] Leber, P.D. and Davis, C.S. (1998) Threats to the validity of clinical trials employing enrichment strategies for sample selection. Control Clin Trials, 19, 178-187.

[40] Welty, G., Klinge, U., Klosterhalfen, B., Kasperk, R. and Schumpelick, V. (2001) Functional impairment and complaints following incisional hernia repair with different polypropylene meshes. Hernia, 5, 142-147.

[41] Sugerman, H.J., Kellum Jr., J.M., Reines, H.D., DeMaria, E.J., Newsome, H.H. and Lowry, J.W. (1996) Greater risk of incisional hernia with morbidly obese than steroid-dependent patients and low recurrence with prefascial polypropylene mesh. Am J Surg, 171, 80-84.

[42] Balen, E.M., Diez-Caballero, A., Hernandez-Lizoain, J. L., Pardo, F., Torramade, J.R., Regueira, F.M. and Cienfuegos, J.A. (1998) Repair of ventral hernias with expanded polytetrafluoroethylene patch. $\mathrm{Br} J$ Surg, 85, $1415-1418$.

[43] Adloff, M. and Arnaud, J.P. (1987) Surgical management of large incisional hernias by an intraperitoneal Mersilene mesh and an aponeurotic graft. Surg Gynecol Obstet, 165, 204-206. 